\title{
Cultural similarities and specificities of finger counting and montring: evidence from Amazon Tsimane' people
}

Krzysztof Cipora ${ }^{1,2,3 *}$, Venera Gashaj ${ }^{2,4}$, Annabel S. Gridley ${ }^{1}$, Mojtaba Soltanlou ${ }^{2,5}, \&$ HansChristoph Nuerk ${ }^{2,3}$

${ }^{1}$ Centre for Mathematical Cognition, Loughborough University, United Kingdom

${ }^{2}$ Department of Psychology, University of Tuebingen, Tuebingen, Germany

${ }^{3}$ LEAD Graduate School \& Research Network, University of Tuebingen, Tuebingen, Germany

${ }^{4}$ Learning Sciences and Higher Education, Swiss Federal Institute of Technology (ETH) Zurich, Zurich, Switzerland

${ }^{5}$ School of Psychology, University of Surrey, Guildford, UK

* Corresponding author: Krzysztof Cipora, Centre for Mathematical Cognition, Loughborough University, LE11 3TU. E-mail: k.cipora@1boro.ac.uk 


\begin{abstract}
Numerical Cognition might be embodied, that is bound to what our bodies are able to do. This claim is supported by the observation that potentially due to our shared biology, finger counting is prevalent among variety of cultures. Differences in finger counting are apparent even within Western cultures. Relatively few indigenous cultures have been systematically analyzed in terms of traditional finger counting and montring (i.e., communicating numbers with fingers) routines. Even fewer studies used the same protocols across cultures, allowing for a systematic comparison of indigenous and Western finger counting routines. We analyze the finger counting and montring routines of Tsimane' $(\mathrm{N}=121)$, an indiginous people living in the Bolivian Amazon rainforest, depending on handedness, education level, and exposure to mainstream, industrialised Bolivian culture. Tsimane' routines are compared with those of German and British participants. Tsimane' reveal a greater variation in finger counting and montring routines, which seems to be modified by their education level. We outline a framework on how different factors such as handedness and reading direction might affect cross-cultural and within-cultural variation in finger counting.
\end{abstract}

179 Words

Keywords: embodied numerical cognition, finger counting, finger montring, Amazon Tsimane', cross-cultural 


\section{Introduction}

\section{Finger counting and montring}

Fingers are the most natural tool for counting (Andres \& Pesenti, 2015; Fischer, Kaufmann, \& Domahs, 2012) and using them for that purpose has been prevalent among humans across millennia. According to archaeological data, the first evidence found for using fingers to count is 27 thousand years old (see Göbel, Shaki, \& Fischer, 2011; Roullion, 2006). Some other authors claim that there is evidence for an even earlier use, over 30 thousand years ago (Wiese, 2003). Finger counting has been prevalent across different cultures and communities (Butterworth, 1999; Ifrah, 1985), and it seems to have played a role in shaping how we think of numbers: Most cultures are using a base-10 system, which probably originates from the use of fingers. In many languages, several words for numbers are directly associated with words referring to hands or fingers. For instance, the words five and fist have the same roots in several languages, including English (Ifrah, 1985)

- fünf [five] and Faust [fist] in German,

- pięć [five] and pięść [fist] in Polish,

- pet [five] and prst [finger] in Bosnian-Croatian-Montenegrin-Serbian,

- $\quad p a n j[$ five] and panjeh [hand] in Farsi,

- pesë [five] in Albanian,

- cinque [five] in French and Italian (pronounced differently),

- cinco Spanish and Portuguese (pronounced differently),

Albanian, French/Italian, and Spanish/Portuguese derived from Proto-IndoEuropean $* \mathrm{pn}_{\mathrm{o}} \mathrm{k}^{\mathrm{w}}$-sti-s and/or Proto-Italic $* \mathrm{k}^{\mathrm{w}} \mathrm{enk}^{\mathrm{w}} \mathrm{e}$, [fist] may go back to a verbal stem * penk $^{\mathrm{w}}$ [to take in hand, to handle] (Sihler, 2008).

In several African languages, e.g., Zulu or Sotho most of the number words are closely related to finger counting (Zaslavsky, 1999) and the same is true for several native languages of America (Closs, 1996).

However, the role of fingers in the development of mathematics should not only be considered in a historical / cultural perspective but also in the ontogenetical perspective of the acquisition of numerical skills by modern humans (Moeller, Martignon, Wessolowski, Engel, \& Nuerk, 2011 for a review).

Use of fingers to handle numbers seems not to be just a co-incidental development, but rather to be playing a functional role, which has been explored in the studies of embodied numerical cognition (see Andres, Olivier, \& Badets, 2008; Di Luca \& Pesenti, 2011; Gashaj, 
Oberer, Mast, \& Roebers, 2019; Morrissey, Liu, Kang, Hallett, \& Wang, 2016; Roesch \& Moeller, 2015). At the same time, studies testing congentially blind children question the natural occurrence and functional role of finger counting for development of numerical representation (Crollen, Mahe, Collignon, \& Seron, 2011; Crollen, Seron, \& Noël, 2011), so the functional role of finger counting is still a subject of a debate.

Many educated adults in Western societies still use fingers for supporting calculations (e.g., counting working days before my vacation starts, counting syllables in a sentence) and communicating numbers to others, i.e., finger montring; for instance, spreading two fingers in the bar whilst saying "two beers please!” (Hohol, Wołoszyn, Nuerk, \& Cipora, 2018; Lucidi \& Thevenot, 2014; Pika, Nicoladis, \& Marentette, 2009). A study among Polish university students (Hohol et al., 2018) shows that around 50\% of participants declares to often or very often use fingers when presenting arguments, and listing elements, and around $40 \%$ declares using fingers for calendar calculations. Lucidi and Thevenot (2014) report that all their participants were using fingers to count syllables in a phrase. About $30 \%$ of the participants tested by Hohol et al. (2018) declares using their fingers often or very often to communicate numbers.

Crucially, the way of communicating numbers with fingers might differ from the way of counting: it is not always the case that demonstrating a number reflects a "snapshot" of the counting sequence. For instance, people in some cultures communicate number three by extending index, middle and ring fingers, while they extend thumb, index, and middle fingers for finger counting (Pika et al., 2009). Moreover, people engage in finger counting mostly for themselves, whereas the goal of finger montring is to communicate the number to other people (Wasner, Moeller, Fischer, \& Nuerk, 2015).

Despite the wide prevalence of using fingers for handling numbers, there are considerable cultural differences in how specifically finger counting and montring are performed. Current literature provides a relatively broad account of finger counting and montring routines in several countries and cultures (e.g. Bender \& Beller, 2011, 2012). Even when considering simple, one-dimensional systems, where numbers are linked to fingers based on one-to-one correspondence (see Bender \& Beller, 2011, for a classification of other body part counting systems; 2012), there is still some space for variation in finger counting: (a) whether the palm is turned toward oneself or toward others, (b) whether the fingers are extended or bent, (c) whether the starting hand is right or left, (d) whether the switch between 
hands is based on anatomical symmetry or spatial continuation, (e) which finger starts the sequence. In all these areas variation has been observed both between and within cultures.

In most Western cultures finger counting starts with closed palms and the extension of the fingers (e.g., Hohol et al., 2018), while in Japan the counting starts with an open palm and fingers are bent successively (Nishiyama, 2013). Another aspect is that in most European cultures people count with their palms facing toward oneself. However, this aspect of finger counting has not been thoroughly investigated yet.

One of the most thoroughly investigated aspects is the starting hand, which according to early claims follows reading/writing direction (Zago \& Badets, 2016). Consequently, one could expect that a majority of Western participants count from left to right, while right-to-left readers (e.g., Arab, Farsi, \& Hebrew speakers) should more often start with their right hand (see Lindemann, Alipour, \& Fischer, 2011). However, later studies have shown that even within Western cultures variability exists: there are more left starters in the UK (e.g., Fischer, 2008), while in Belgium and France the majority starts with their right hand (e.g., Sato \& Lalain, 2008) and in Poland there is a relatively equal split between left-starters and rightstarters (Hohol et al., 2018). This shows that even within a single culture there might still be some variation (see also Morrissey et al., 2016). Another factor that should be considered when it comes to the starting hand is participant's handedness with left-handers being more likely to start with their left hand, while in case of right-handers there is a more equal share of left- and right-starters (Hohol et al., 2018; Morrissey et al., 2016)

After reaching number five, one typically needs to switch hands, and can continue counting with the other hand either by anatomical symmetry (e.g., start with the same finger of the other hand), or by spatial order (continue with the spatially closest finger). The vast majority of Western participants uses anatomically symmetric continuation (Hohol et al., 2018; Lindemann et al., 2011), while the prevalence of the spatial continuation is slightly higher among Middle Eastern participants (Lindemann et al., 2011). Nevertheless, in both groups the anatomical symmetry remained more prevalent.

In most Western cultures participants start the counting sequence with their thumb (Hohol et al., 2018) but there are several cultures such as in the Middle East where people start counting with their pinkie (Lindemann et al., 2011) or as it is common in China - starting from their index finger, then middle finger, ring finger, pinkie, and ending with the thumb (Domahs, Moeller, Huber, Willmes, \& Nuerk, 2010).

When it comes to montring, systematics data is more scarce (Di Luca, Granà, Semenza, Seron, \& Pesenti, 2006; Di Luca \& Pesenti, 2008, 2011). However, even within Western 
cultures there is a variation in how finger montring is executed, and how it differs from finger counting. For instance, French Canadians tend to use their thumb to represent numeral 1 when counting to 10 , but when asked to gesture number 1 only they use their index finger. At the same time, Germans would rather use their thumb for montring 1 (Pika et al., 2009). The observed discrepancy between finger counting and montring patterns and cultural variations in finger montring call for testing both counting and montring while investigating how fingers and numbers are linked in different cultures.

The literature on finger counting mostly covers Western Europe, North America, to some extent the Middle East (but it is mostly represented by Iran), and East Asia: China and Japan. In addition to that, there is a number of reports of indigenous tribes showing very specific and elaborated finger / body part counting systems (see Bender \& Beller, 2012 for a review; Closs, 1996; Overmann, 2021; Zaslavsky, 1999). Other than that, non-industrialized cultures remain massively underinvestigated, which reflects the sample representativeness problems in psychology (Henrich, Heine, \& Norenzayan, 2010). For this reason, it is important to widen the literature coverage of as many indigenous cultures as possible, not only the ones, which present apparent and very atypical patterns. What we find particularly important is ensuring (as much as possible) that one can directly compare the results from indigenous samples with the ones from Western cultures.

One might expect that because of being relatively isolated from globalization (e.g., presence of global mass-media culture, industrialization), indigineous cultures might offer novel insights into the nature of finger counting and montring and its variety among humans. For instance, while several cultures developed very elaborate body part counting systems (see Bender \& Beller, 2012; Saxe \& Esmonde, 2012), there is evidence for cultures not using fingers and other body parts to communicate numbers at all (e.g., Butterworth, Reeve, \& Reynolds, 2011; Everett, 2017). This shows that finger usage may not be taken for granted in every culture and population.

\section{Tsimane' people}

Tsimane' people are an indigenous, non-industrialized group living in the Bolivian Amazon rainforest. They are a population of about 8000 people (Piantadosi, Jara-Ettinger, \& Gibson, 2014). Their lifestyle differs considerably from the industrialized cultures: they engage in demanding daily physical activity, including hunting-gathering and subsistence farming (Godoy \& Jacobson, 1999; Godoy, Jacobson, \& Wilkie, 1998; Huanca, 2008). Moreover, they 
differ in respect to several psychological variables, which include visual perception, health locus of control (externalized in Tsimane' i.e., they are more likely to attribute their health and its changes to the external factors, including chance or the intervention of gods/spirits than to their own health-related behaviors), or personality structure; Tsimane' personality is better described by two dimensions of leadership/prosociality and industriousness rather than the supposedly universal big five model of extroversion, neuroticism, openness to experience, conscientiousness, and agreeableness (van der Linden et al., 2018).

This population has also been found to have no or poor knowledge of arithmetic, which could be due to adults receiving no formal education, as early education for Tsimane' children is not prioritized (Piantadosi et al., 2014). Technically, each community has its own school, which is led by a teacher, who is nominated by the community, and who does not receive formal teacher training. Schooling is not mandatory, and there is no strict division by grades, meaning that the same curriculum is taught every year. Unlike in industrialized cultures, children do not have access to TV or educational toys to support their learning. The lack of mandatory education in practice leads to a very large heterogeneity within communities when it comes to numeracy and literacy (Piantadosi et al., 2014). Less than $10 \%$ of their callory intake comes from purchased products, so their daily experience with money is also relatively limited (Blackwell et al., 2017). Interestingly, from a perspective of acquisition of number concepts, Tsimane' children follow the same pathway as their peers in industrialized countries, but with a delayed and more variable timeline (Jara-Ettinger, Gibson, Kidd, \& Piantadosi, 2016).

\section{Finger counting in Tsimane' initial investigation}

Before formal data collection with Tsimane' participants, we asked our translators (bilingual Tsimane') on whether Tsimane' count with their fingers and whether they would understand the task. We learned that finger counting is indeed widespread. However, their finger counting routines do not go beyond the simple one-to-one correspondence (i.e., can be classified as simple, one-dimensional system). Tsimane' language is using a base-10 system, the structure of multi-digit numbers can be considered transparent and reflects the place-value structure. At the same time there are some hints that they might have used a quinary (base-5) system or a mixture of quinary and base-10 system ${ }^{1}$. This might suggest the role of finger

\footnotetext{
${ }^{1}$ https://lingweb.eva.mpg.de/channumerals/Tsimane.htm
} 
counting in formation on Tsimane' language related to numbers, similar to other languages where parallels between number words for five and names for hand/palm have been described. Moreover, we asked our translators whether there are any similarities and explicit references between number words and words referring to hand, palm, fist etc. They could not identify such similarities. While Tsimane' mostly count in their language when it comes to small numbers, numbers larger than 50 are rarely used in Tsimane' language, and if they use them, they rather use Spanish number words.

\section{Objectives of the present study}

In the current study, we investigated finger counting and finger montring in Tsimane' people. To further verify the reading/writing direction as a factor influencing finger counting, we look at whether the finger counting pattern being in line with the typical reading and writing direction is more prevalent among individuals, who are more exposed to schooling and the industrialised Bolivian Spanish-language community. We also aimed at providing quantitative data on prevalence of different finger counting and montring routines in Tsimane'. By looking at these patterns, we aimed at further testing independence of finger counting and montring. Additionally we compare Tsimane' to German and British control groups tested using the same protocol. These control groups, despite both being Western European have been documented to differ from each other, especially in finger montring (Pika et al., 2009).

\section{Method}

The research in Bolivia was conducted in the summer of 2018 in several villages near the city of San Borja, Bolivia. We worked with the local research center, the Centro Boliviano de Investigación y de Desarollo Socio Integral (CBIDSI), working with its members T. Huanca and R. Godoy. CBIDSI coordinated logistics, participant recruitment and native translators. They also served as experts in Tsimane' culture. Our studies were approved by the Gran Consejo Tsimane' (Tsimane' Grand Council) and by the MIT's Committee on the Use of Humans as Experimental Subjects (COUHES). Participants were compensated with gifts worth around \$20 for this and unrelated studies that they participated in (taking under 40 mins of their time). The gifts were designated by T. Huanca at the CBIDSI.

Research in Germany was conducted in spring semester of 2019 while in the UK the data were collected in spring semester 2021. 


\section{Participants}

\section{Tsimane’ Sample.}

A total of 121 Tsimane' (60 female, 53 male, in case of eight participants gender was not recorded during the data collection), were tested $(M=34.54, S D=15.14$, range $=15-83$ years $)$. Tsimane' participants varied greatly in terms of their education, ranging from no schooling at all to 15 years of schooling $(M=3.78, \mathrm{SD}=3.99$ years $)$. The exact data on number of years of schooling is available in the shared datafile. For the analysis, we classified the participants into three groups: (1) no schooling, $\mathrm{n}=30$; (2) 1 to 5 years of schooling $\mathrm{n}=55$; (3) more than 5 years of schooling, $\mathrm{n}=28^{2}$. Participants also differed with regards to the extent they travelled to neighboring towns, and the extent of contact they had with Bolivian, Spanish speaking society. On average, they travelled $3.89(S D=5.29)$ times per month (ranging from 0.08 to 42.9 times per month). Note that participants provided frequency and unit (per week / month / year) and the frequency was scaled to number of travel / month, thus the minimum of 0.08 corresponds to 1 travel / year etc.). The frequency data is reported in the shared datafile. For the purpose of this analysis, we categorized participants into two groups, of those travelling less than twice a month $(n=47)$, and those travelling twice a month and more $(n=66)^{3}$. The data on age, sex, education and travel frequency was not available for eight individuals.

German Sample. A total of 60 (41 female, 19 male) German adults were tested. Fiftyseven of which were students at the University of Tuebingen, while three were completing a Voluntary Social Year $(M=23.5, S D=3.1$, range $=18-31$ years $)$. Participants were compensated with course credit or $4 €$. The study was approved by the ethics committee of the Department of Psychology at the University of Tuebingen, Germany.

British Sample. A total of 61 (34 female, 27 male) British adults were tested. Fifty of them were university students, eight were employees, and three were retired $(M=28.4, S D=$ 16.7 , range $=18-85$ years). Participants performed only tasks reported here and were not compensated for their participation. The study was approved by the Ethics Review SubCommittee of Loughborough University.

\footnotetext{
${ }^{2}$ Gibson, Jara-Ettinger, Levy, and Piantadosi (2017) found that the relation between education and other numerical tasks in Tsimane' disappears in participants having received between 5 and 6 years of education. Moreover, this threshold allowed us to keep the group sizes relatively equal. However, we acknowledge that others might have used some other threshold that might affect between-study comparisons. For instance, Ferrigno, Jara-Ettinger, Piantadosi, and Cantlon (2017) used a binary split for more than vs. less than one year of education.

${ }^{3}$ Again, this cut-off is arbitrary. It allowed to form relatively equal groups and seemed intuitively reasonable to quantify "frequent" travel and engagement with the mainstream Bolivian Spanish-language community. Full data on travel frequency is available online and allows testing other cut-offs.
} 


\section{Materials and Procedure}

This study was part of a set of tasks looking into different aspects of magnitude representation in Tsimane' people. Other administered tasks were unrelated to finger counting and thus out of scope for the present paper.

\section{Session plan}

Each session started with obtaining informed consent: verbal in case of Tsimane', written in case of German (paper form) and British (online questionnaire). Then demographic data was collected. Subsequently, the handedness tasks were administered, followed by finger montring, and finger counting tasks. The German and Tsimane' participants completed other tasks which are not reported here, whereas the session ended after the finger counting task for the British participants.

Tsimane' group. Tasks were administered under supervision of the first author by a trained translator, who was Tsimane'-Spanish bilingual, and responses were coded by the first author. The translator was administering tasks in Tsimane' based on written protocol translated into Spanish by trained psychologists who are English-Spanish bilinguals. The translators were trained in the administration of tasks prior to data collection.

German group. Data was collected, administered, and coded by a native German speaker. The original protocol was translated into German by a trained psychologist who is a native German speaker and fluent in English.

British group. Tasks were administered and responses coded by a native English speaker. Data was collected online by means of a video call with the experimenter (in-person data collection was not possible due to the COVID-19 pandemic). Prior to the experiment, the camera was adjusted so that the experimenter could see the upper trunk of the participant and their hands. Additionally, it was checked whether the image was mirror flipped.

\section{Handedness assessment}

As several Tsimane' participants were illiterate and were unfamiliar with several activities referred to in formal handedness questionnaires, we used simple performance measures. First, the participants were given a piece of paper and a pencil. The pencil was placed centrally on a tabletop on a distal side and parallel to paper's distal edge. Participants were then asked to draw three horizontal lines (task 1a). Subsequently, they were given (put centrally on the tabletop) a ball made of paper and were asked to throw it (task 2a). Finally, they were given another piece of paper and a pencil (as in task 1a) and were asked to draw a circle (task $3 a)$. 
The hand they were using was recorded for each of these tasks separately. The procedure was the same for German participants. For the British participants due to the online nature of the experiment (video call), the tasks were adjusted. First, they were asked, which hand they are using to throw a ball (task 1b), to write (task 2b), and to brush their teeth (task $3 b$ ). The hand they were using was recorded for each of these tasks separately.

In sum, there was handedness data from three situations for each participant. Participants were classified as right-handers if they performed all three tasks with their right hand, as left-handers if they performed all tasks with their left hand, and as non-right-handers if they performed some of the tasks with the right and some of them with the left hand.

\section{Finger montring}

This task was the same for all groups. Participants were asked to show numbers 1, 8, 6, 4, 9 and 3 with their fingers. The order of numbers was fixed for all participants. The fingers being used were recorded by the experimenter.

\section{Finger counting}

This task was the same for all groups. Participants were asked to count aloud and by using their fingers up to 10. The starting hand, starting finger, as well as which finger of the other hand they continued the counting on were recorded.

During data collection in Tsimane', it occurred that several participants were bending their fingers while counting (unlike the typical European technique of extending them). Only after spotting that we started recording it systematically, so this data is only available for a limited number of Tsimane' participants $(n=23)$. However, it is available for German (except 1 participant) and British participants who were tested afterwards. The counting technique was categorized as "extend" (participant starts with closed palm and extends fingers), "bend" (participant starts with open palm and bends fingers), or "keep out" (participant keep the palm open and uses another hand to touch the fingers being counted).

\section{Data analysis}

In the first step we investigated cultural differences in finger counting patterns, and its relation to handedness. Furthermore, we looked into Tsimane' to see whether their finger counting routines differed depending on their schooling and frequency of travel to the town. Eventually, we looked at within participant similarity of finger pattern being used to for counting and montring number 1 as using this number can give an impression on how potentially observed group-level differences between finger counting and montring correspond to within-subject similarities of patterns used for counting and montring a given number. Due to the nature of 
the data (categorical), non-parametric tests were used $\left(\chi^{2}\right)$ and its Bayesian equivalents so that both evidence for between group difference or a lack thereof could be reported. Analyses were conducted in R language, using packages dplyr (Wickham, François, Henry, \& Müller, 2020), jmv (Selker, Love, \& Dropmann, 2020), and jsq (Love, Selker, \& Dropmann, 2021). Raw data and analysis scripts are available at https://osf.io/wj582/?view_only=755618307ce6493fb74cf7d47db6eda0 .

\section{Results}

\section{Between group comparisons}

\section{Handedness}

In all three groups, the majority (87\%) of participants were right-handed. Proportions of right, left-, non-right-handers did not differ between groups, $\chi^{2}(2, N=242)=3.72, p=.445, B F_{01}=$ 51.44 (cf. Table 1).

Table 1: Handedness and Starting Hand

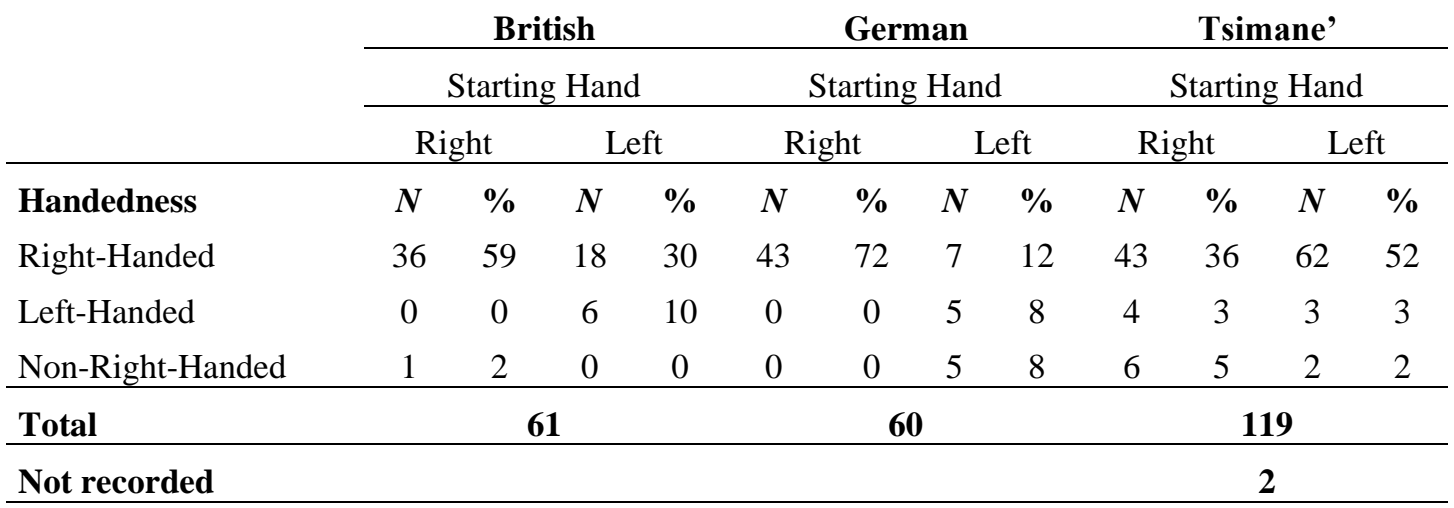

Note. Not recorded refers to the participants that were not available for this particular observation.

\section{Finger counting}

Starting hand. Starting hand data was not available for one Tsimane' participant. There was an evident between group difference as regards to the starting hand, $\chi^{2}(2, N=241)=$ 13.22, $p=.001, B F_{10}=88.47$. While the majority of Tsimane' started finger counting with their left hand, the majority of German and British participants started with their right hand (Table 1).

Starting hand by handedness. Results are summarized in Table 1. In Tsimane', handedness was not associated with the starting hand, $\chi^{2}(2, N=120)=30.35, p=.135, B F_{01}$ 
$=1.94$, but in the light of Bayesian analysis this result remains inconclusive. Also, in terms of descriptive statistics the split between right-, non-right- and left-handers was relatively similar with left- and right-starters.

The effect was evident both in German, $\chi^{2}(2, N=60)=30.35, p<.001, B F_{10}=$ 90365.17, and British, $\chi^{2}(2, N=61)=10.72, p=.005, B F_{10}=14.29$, samples: none of the lefthanders started finger counting with their right hand. In both groups a majority of right-handers started with their right hand. All non-right handers in the German sample started with their left hand too, in the British sample there was only one non-right-hander who started from their right hand.

Table 2: Finger Counting Patterns and Techniques

\begin{tabular}{crrrrr}
\multicolumn{2}{c}{ British } & \multicolumn{2}{c}{ German } & \multicolumn{2}{c}{ Tsimane' } \\
\hline$N$ & $\%$ & $N$ & $\%$ & $N$ & $\%$ \\
\hline
\end{tabular}

\section{Counting pattern}

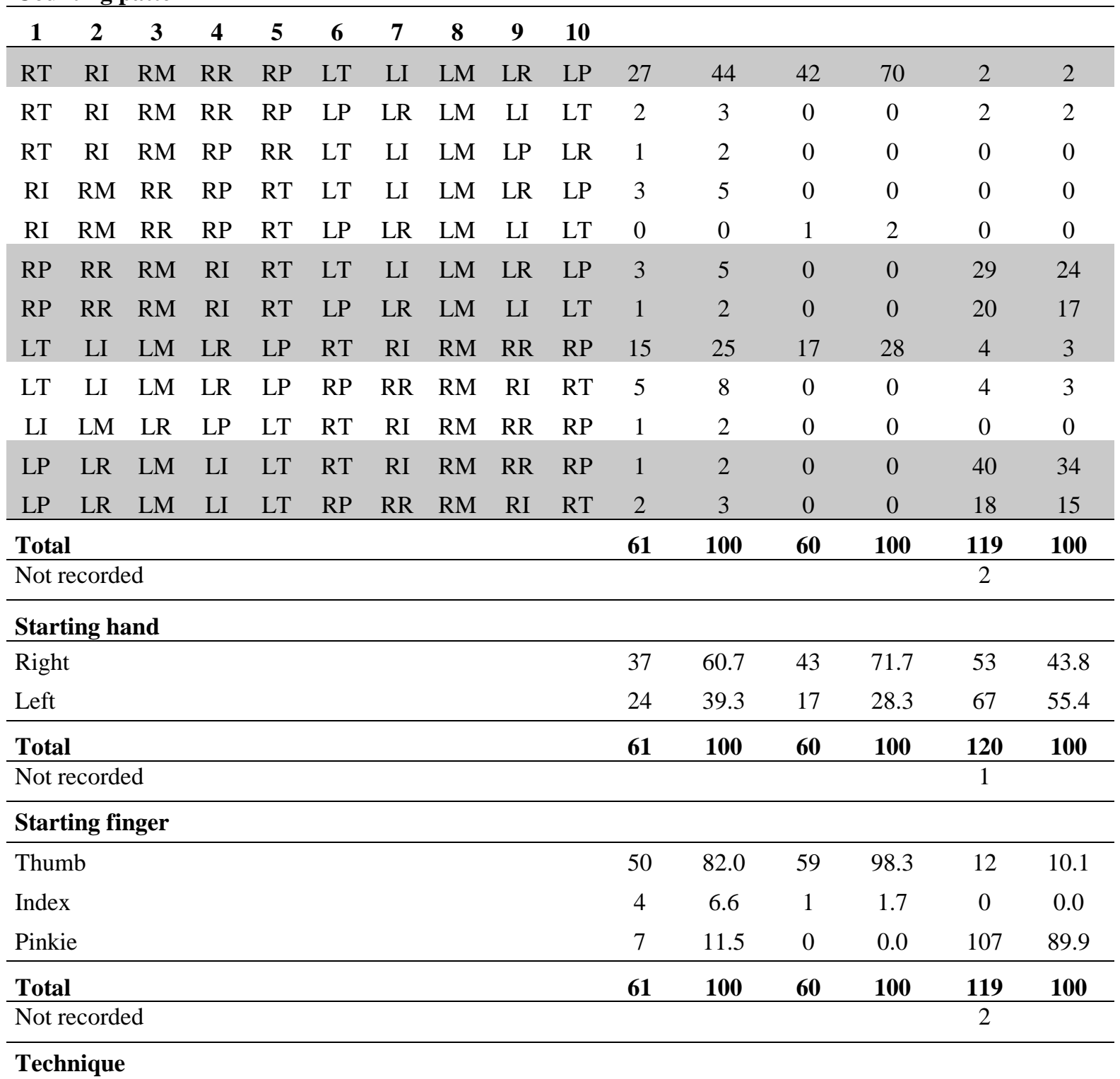




\begin{tabular}{lcccccc} 
Extend & 53 & 86.9 & 59 & 100 & 12 & 52.2 \\
Keep out & 8 & 13.1 & 0 & 0 & 0 & 0.0 \\
Bend in & 0 & 0.0 & 0 & 0 & 11 & 47.8 \\
\hline Total & $\mathbf{6 1}$ & $\mathbf{1 0 0}$ & $\mathbf{5 9}$ & $\mathbf{1 0 0}$ & $\mathbf{2 3}$ & $\mathbf{1 0 0}$ \\
\hline Not recorded & & & 1 & & 99 \\
\hline
\end{tabular}

Note. Counting pattern: The hand ( $\mathrm{L}$ and $\mathrm{R}$ for left and right hand respectively) and finger $(\mathrm{T}=\mathrm{thumb}, \mathrm{I}=$ index, $\mathrm{M}=$ middle, $\mathrm{R}=$ ring, $\mathrm{P}=$ pinkie) used to represent the specific number in the counting sequence (top row). The most prevalent (frequency $>20 \%$ in any group) patterns are highlighted.

Finger counting patterns. A summary of finger counting patterns is presented in the upper part of Table 2. In total we observed 12 different patterns. All participants used both hands to count up to 10 . In the following, by "continuation" we mean the finger of the second hand being used to count 6 . There was a remarkable difference in finger counting patterns between Tsimane', German and British participants; In case of Tsimane', the most prevalent pattern was starting with their left pinkie and continuing with the right thumb. Other prevalent patterns were starting with the left pinkie and continuing with the right pinkie or starting with the right pinkie and continuing with the left pinkie or left thumb. For German and British participants, starting with a thumb and continuing with the thumb of the other hand were by far the most prevalent strategies. Interestingly, also the continuation of the finger counting sequence (i.e., which finger was used when counting six) differed between groups; While the majority of German and British participants were using their thumb (i.e., following anatomical symmetry), Tsimane' showed more variation. In case of participants starting with their right pinkie, the continuation with the left thumb was slightly more prevalent than continuation with the left pinkie. In case of individuals starting with the left pinkie, continuation with right thumb was way more prevalent than the continuation with the right pinkie. This means that while anatomical symmetry was present in the majority of German and British participants, it was observed only in a minority of Tsimane'. The latter in fact followed neither symmetry nor spatial continuation.

The less prevalent patterns, observed in fewer than $20 \%$ of each sample were: (1) starting with a right thumb and continuing with a left pinkie, (2) starting with a right thumb, continuing with a left thumb, using left pinkie as 9 and left ring as 10, (3) starting with right index, 5 with right thumb, and then continuing from left thumb, (4) starting with right index, 5 with right thumb, then from left pinkie, (5) starting with left thumb, and continuation from right pinkie, (6) starting with left index, 5 with left thumb, continuation with right thumb.

Starting finger. Data on starting finger are summarized in the bottom part of Table 2. Data from two Tsimane' participants were not available. There was an evident between group 
difference with regards to the starting finger, $\chi^{2}(4, N=240)=176.39, p<.001, B F_{10}>10^{42}$. While the majority of Tsimane' started with their pinkie, most German and British participants started with their thumb (see also Figure 1).

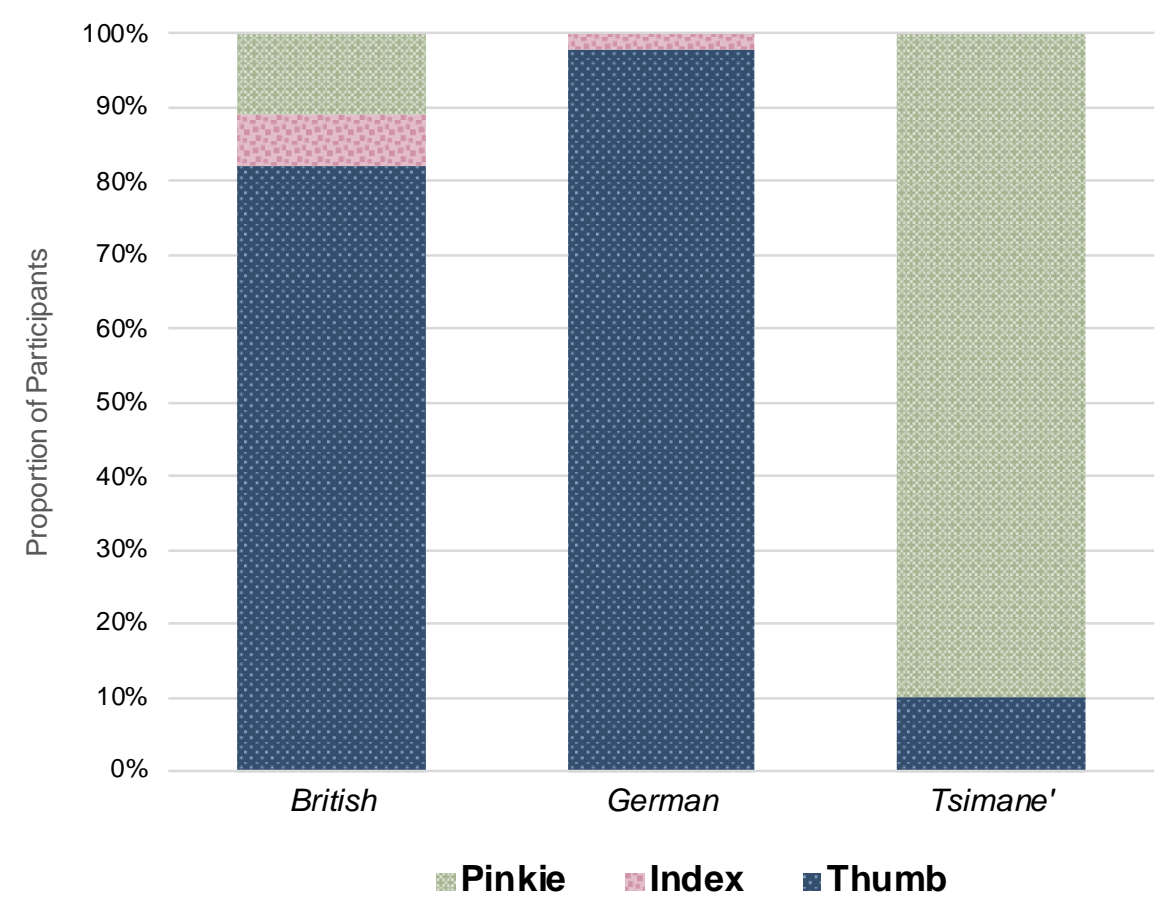

Figure1. Starting finger used when counting to 10

Finger counting technique. The data on whether the participants were counting by bending or extending their fingers was only available for a limited number of Tsimane' (cf. Table 2). Nevertheless, the difference between groups was evident, $\chi^{2}(4, N=143)=75.11, p$ $<.001, B F_{10}>10^{8}$. While about half of Tsimane' started with an open palm and bent their fingers when counting, the other half extended their fingers starting with a closed palm. The latter was the only technique observed in German participants and was by far a majority in British participants. The second technique observed in British participants was the "kept out" technique, that is the palm remained open and the participant was using a finger of the other hand as a pointer touching the counted fingers.

\section{Finger Montring}

Number 1. One Tsimane' participant showed this number incorrectly. Other than that, there were seven different patterns of how participants showed number one (cf. Table 3). There was a noticeable between group difference, $\chi^{2}(12, N=241)=120.65, p<.001, B F_{10}>10^{17}$. Most 
prevalent patterns in Tsimane' were lifting right, left index finger, or left pinkie. In German participants the split between right thumb and right index finger was almost equal, while in British the vast majority of participants used their index finger. 
Table 3a. Finger montring techniques by group and number 1-4

\begin{tabular}{|c|c|c|c|c|c|c|c|c|c|c|}
\hline \multirow{3}{*}{ Number } & \multirow{3}{*}{ Technique } & \multicolumn{9}{|c|}{ Group } \\
\hline & & \multirow[t]{2}{*}{$\begin{array}{l}\text { Images of } \\
\text { technique }\end{array}$} & \multicolumn{2}{|c|}{ British } & \multicolumn{2}{|c|}{ German } & \multicolumn{2}{|c|}{ Tsimane' } & \multicolumn{2}{|c|}{$\begin{array}{l}\text { Error in } \\
\text { Tsimane' }\end{array}$} \\
\hline & & & $\mathrm{N}$ & $\%$ & $\mathrm{~N}$ & $\%$ & $\mathrm{~N}$ & $\%$ & $\mathrm{~N}$ & $\%$ \\
\hline \multirow{8}{*}{1} & RT up & tै & 2 & 3.3 & 29 & 48.3 & 6 & 5.0 & \multirow{7}{*}{1} & \multirow{7}{*}{0.8} \\
\hline & RI up & $\theta$ & 50 & 82.0 & 20 & 33.3 & 62 & 51.2 & & \\
\hline & RM up & & 0 & 0.0 & 0 & 0.0 & 1 & 0.8 & & \\
\hline & RP up & & 0 & 0.0 & 0 & 0.0 & 9 & 7.4 & & \\
\hline & LT up & & 0 & 0.0 & 9 & 15.0 & 3 & 2.5 & & \\
\hline & Ll up & & 9 & 14.8 & 2 & 3.3 & 22 & 18.2 & & \\
\hline & LP up & & 0 & 0.0 & 0 & 0.0 & 17 & 14.0 & & \\
\hline & Total & & \multicolumn{2}{|c|}{61} & \multicolumn{2}{|c|}{60} & \multicolumn{2}{|c|}{120} & \multicolumn{2}{|c|}{121} \\
\hline \multirow{8}{*}{3} & RI, RM, RR up & & 40 & 65.6 & 7 & 11.7 & 3 & 2.5 & \multirow{7}{*}{0} & \multirow{7}{*}{0} \\
\hline & RM, RR, RP up & & 2 & 3.3 & 0 & 0.0 & 62 & 51.2 & & \\
\hline & RT, RI, RM up & & 9 & 14.8 & 39 & 65.0 & 4 & 3.3 & & \\
\hline & LI, LM, LR up & & 7 & 11.5 & 0 & 0.0 & 2 & 1.7 & & \\
\hline & LM, LR, LP up & & 1 & 1.6 & 0 & 0.0 & 49 & 40.5 & & \\
\hline & LT, LI, LM up & & 1 & 1.6 & 14 & 23.3 & 1 & 0.8 & & \\
\hline & LR, LI, RT up & & 1 & 1.6 & 0 & 0.0 & 0 & 0.0 & & \\
\hline & Total & & \multicolumn{2}{|c|}{61} & \multicolumn{2}{|c|}{60} & \multicolumn{2}{|c|}{121} & \multicolumn{2}{|c|}{121} \\
\hline \multirow{6}{*}{4} & RT down & & 48 & 78.7 & 37 & 61.7 & 60 & 49.6 & \multirow{5}{*}{1} & \multirow{5}{*}{0.8} \\
\hline & RP down & & 1 & 1.6 & 6 & 10.0 & 3 & 2.5 & & \\
\hline & & & & & & & & & & \\
\hline & LT down & & 12 & 19.7 & 16 & 26.7 & 53 & 43.8 & & \\
\hline & LP down & & 0 & 0.0 & 1 & 1.7 & 4 & 3.3 & & \\
\hline & Total & & \multicolumn{2}{|c|}{61} & \multicolumn{2}{|c|}{60} & & & \multicolumn{2}{|c|}{121} \\
\hline
\end{tabular}

Note. Technique: The letters $R$ and $L$ mean left or right hand and $T=$ thumb, $I=$ index, $M=$ middle, $R=$ ring, $P=$ pinkie finger used. 
Table $3 b$. Finger montring techniques by group and number 6-9

\begin{tabular}{|c|c|c|c|c|c|c|c|c|c|c|}
\hline \multirow{3}{*}{ Number } & \multirow{3}{*}{ Technique } & \multicolumn{9}{|c|}{ Group } \\
\hline & & \multirow[t]{2}{*}{$\begin{array}{l}\text { Images of } \\
\text { technique }\end{array}$} & \multicolumn{2}{|c|}{ British } & \multicolumn{2}{|c|}{ German } & \multicolumn{2}{|c|}{ Tsimane' } & \multicolumn{2}{|c|}{$\begin{array}{l}\text { Error in } \\
\text { Tsimane' }\end{array}$} \\
\hline & & & $\mathrm{N}$ & $\%$ & $\mathrm{~N}$ & $\%$ & $\mathrm{~N}$ & $\%$ & $\mathrm{~N}$ & $\%$ \\
\hline \multirow{8}{*}{6} & RT up & & 23 & 37.7 & 26 & 43.3 & 42 & 34.7 & \multirow{7}{*}{9} & \multirow{7}{*}{7.4} \\
\hline & RI up & & 6 & 9.8 & 1 & 1.7 & 5 & 4.7 & & \\
\hline & RP up & & 1 & 1.6 & 0 & 0.0 & 8 & 7.5 & & \\
\hline & LT up & & 24 & 39.3 & 31 & 51.7 & 38 & 31.4 & & \\
\hline & LI up & & 3 & 4.9 & 2 & 3.3 & 8 & 6.6 & & \\
\hline & LP up & & 4 & 6.6 & 0 & 0.0 & 10 & 9.4 & & \\
\hline & $\begin{array}{l}\text { LT, LI, LM, RT, RI, } \\
\text { RM up }\end{array}$ & & 0 & 0.0 & 0 & 0.0 & 1 & 0.9 & & \\
\hline & Total & & \multicolumn{2}{|c|}{61} & \multicolumn{2}{|c|}{60} & \multicolumn{2}{|c|}{112} & \multicolumn{2}{|c|}{121} \\
\hline \multirow{8}{*}{8} & $\mathrm{RT}$ and RP down & & 12 & 19.7 & 1 & 1.7 & 6 & 5.0 & \multirow{7}{*}{12} & \multirow{7}{*}{9.5} \\
\hline & $\mathrm{RT}$ and $\mathrm{RI}$ down & & 3 & 4.9 & 0 & 0.0 & 14 & 11.6 & & \\
\hline & RR and RP down & & 7 & 11.5 & 28 & 46.7 & 31 & 25.6 & & \\
\hline & $\mathrm{LT}$ and RT down & & 10 & 16.4 & 0 & 0.0 & 10 & 8.3 & & \\
\hline & LT and LP down & & 2 & 3.3 & 0 & 0.0 & 5 & 4.1 & & \\
\hline & LT and LI down & & 6 & 9.8 & 1 & 1.7 & 13 & 10.7 & & \\
\hline & $L R$ and $L P$ down & & 21 & 34.4 & 30 & 50.0 & 30 & 24.8 & & \\
\hline & Total & & \multicolumn{2}{|c|}{61} & \multicolumn{2}{|c|}{60} & \multicolumn{2}{|c|}{109} & \multicolumn{2}{|c|}{121} \\
\hline \multirow{5}{*}{9} & RT down & & 32 & 52.5 & 22 & 36.7 & 29 & 24.0 & \multirow{4}{*}{17} & \multirow{4}{*}{14} \\
\hline & RP down & & 3 & 4.9 & 5 & 8.3 & 20 & 16.5 & & \\
\hline & LT down & & 20 & 32.8 & 28 & 46.7 & 30 & 24.8 & & \\
\hline & LP down & & 6 & 9.8 & 5 & 8.3 & 25 & 20.7 & & \\
\hline & Total & & & & & & & & & \\
\hline
\end{tabular}

Note.Technique: The letters $R$ and $L$ mean left or right hand and $T=$ thumb, $l=$ index, $M=$ middle, $R=$ ring, $P=$ pinkie finger $u$ sed. 
Number 3. All Tsimane' participants showed this number correctly. In total there were seven different patterns of how participants showed number three. There was an evident between group difference, $\chi^{2}(12, N=242)=306.76, p<.001, B F_{10}>10^{59}$. A vast majority of Tsimane' were extending fingers from pinkie to middle of either right (more prevalent) or left hand (slightly less prevalent). Germans most frequently extended thumb, index and middle finger of their right hand (or less frequently their left hand), while the vast majority of British participants extended index, middle, and ring finger of their right hand.

Number 4. One Tsimane' participant showed this number incorrectly. Other than that, there were four different patterns of how participants showed number four. There was a large between group difference, $\chi^{2}(6, N=241)=22.82, p<.001, B F_{10}=11.98$. The most popular patterns across all groups was extended fingers from index to pinkie. Using the right hand was more frequent altogether: slightly more in Tsimane', and way more frequent in German and British.

Number 6. A total of 9 Tsimane' participants showed this number incorrectly. Other than that, there were seven different patterns of how participants showed number six. The between group difference reached significance, but the Bayesian analysis still strongly favored the null hypothesis model, $\chi^{2}(12, N=233)=21.19, p=.048, B F_{01}=8.15$. The most popular patterns were having one hand open and a thumb of the other hand in addition to it.

Number 8. A total of 12 Tsimane' participants showed this number incorrectly. Other than that, there were seven different patterns of how participants showed number eight. There was an evident between group difference, $\chi^{2}(12, N=230)=58.63, p<.001, B F_{10}>10^{9}$. The most popular patterns in Tsimane' were having either left or right ring and pinkie fingers folded. The majority of German participants folded pinkie and ring fingers of either right or left hand. In British participants, the most prevalent pattern was left pinkie and ring fingers folded.

Number 9. A total of 17 Tsimane' participant showed this number incorrectly. Other than that, there were four different patterns of how participants showed number nine. There was a large between group difference, $\chi^{2}(6, N=225)=25.16, p<.001, B F_{10}=1132.59$. In Tsimane' all patterns had roughly equal share. In German and British, patterns with folded left or right thumb were most prevalent.

\section{Analyses within Tsimane'}

\section{Finger counting}


Finger counting in Tsimane' depending on their education and frequency of travel is presented in Table 4. Complete data was available for 112 Tsimane' participants. There was a large between group difference, $\chi^{2}(2, N=112)=8.70, p=.013, B F_{10}=17.77$. The proportion of left-starters increased with increasing number of years of schooling. In case of travel frequency, the effect was not significant, $\chi^{2}(1, N=112)=3.56, p=.059, B F_{10}=2.58$, but seems to suggest that there were more left-starters among those, who travelled to the town more often.

At the same time, starting finger was neither associated with education, $\chi^{2}(2, N=111)$ $=2.67, p=.264, B F_{01}=3.41$, nor with travel frequency, $\chi^{2}(1, N=111)=0.081, p=.776, B F_{01}$ $=3.47$.

Table 4 Finger Counting Pattern, Education, and Travel Frequency

\begin{tabular}{ccccc}
$\begin{array}{c}\text { Educational } \\
\text { Level }\end{array}$ & \multicolumn{2}{c}{$\begin{array}{c}\text { Travel } \\
\text { Frequency }\end{array}$} \\
\hline E1 & E2 & E3 & T1 & T2 \\
\hline
\end{tabular}

\begin{tabular}{|c|c|c|c|c|c|c|c|c|c|c|c|c|c|c|}
\hline Cou & ng p & ern & & & & & & & & E1 & E2 & 3 & T1 & $\mathbf{T} 2$ \\
\hline 1 & 2 & 3 & 4 & 5 & 6 & 7 & 8 & 9 & 10 & & & & & \\
\hline RT & RI & RM & RR & $\mathrm{RP}$ & LT & LI & LM & LR & LP & 1 & 0 & 1 & 0 & 2 \\
\hline RT & RI & $\mathrm{RM}$ & RR & $\mathrm{RP}$ & LP & LR & LM & LI & LT & 0 & 1 & 1 & 2 & 0 \\
\hline RP & RR & RM & RI & RT & LT & LI & LM & LR & LP & 9 & 15 & 3 & 13 & 14 \\
\hline $\mathrm{RP}$ & RR & RM & RI & $\mathrm{RT}$ & LP & LR & LM & LI & $\mathrm{LT}$ & 9 & 7 & 2 & 10 & 8 \\
\hline LT & LI & LM & LR & LP & RT & RI & $\mathrm{RM}$ & $\mathrm{RR}$ & RP & 1 & 2 & 1 & 2 & 2 \\
\hline LT & LI & LM & LR & LP & $\mathrm{RP}$ & RR & $\mathrm{RM}$ & RI & RT & 0 & 1 & 2 & 1 & 2 \\
\hline LP & LR & LM & LI & LT & RT & RI & $\mathrm{RM}$ & $\mathrm{RR}$ & RP & 8 & 19 & 11 & 14 & 24 \\
\hline LP & LR & LM & LI & $\mathrm{LT}$ & RP & RR & $\mathrm{RM}$ & RI & RT & 2 & 8 & 7 & 4 & 13 \\
\hline
\end{tabular}

Total

$\begin{array}{lllll}30 & 53 & 28 & 46 & 65\end{array}$

Note. Educational Level E1 = no schooling; E2 = 1 to 5 years of schooling; E3 = more than 5 years of schooling. Travel Frequency $\mathrm{T} 1=$ less than twice a month; $\mathrm{T} 2=$ twice a month and more. The most prevalent (frequency $>20 \%$ in any group) patterns are highlighted.

\section{Finger montring}

Finger montring in Tsimane' did not differ depending on their education and frequency of travel (cf. Table S1 in Supplementary Material). Incorrect responses were excluded from this analysis and were looked at separately. There were no associations between education or frequency of travel and the way in which participants showed numbers $1,3,4,6$, and 8 . The results were inconclusive for number 9. 
While the frequentist test did not show an association between education and the way participants presented number 9, the Bayesian test suggested that there was a between group difference, $\chi^{2}(6, N=96)=8.58, p=.198, B F_{10}=4.29$. This was most likely driven by the fact that the majority of participants with 1 to 5 years of schooling were folding their left thumb, while the majority of participants with more than 5 years of schooling were folding their right thumb (which to a certain degree reflects the observations for counting, that is, more educated individuals seem to start counting on their left hand). In case of travel frequency there was no such effect.

Errors. Tsimane' participants made a total of 40 finger montring errors, which totals to $5.90 \%$ of all trials. The error rate in the E1 group was $11.67 \%$, in the E2 group it was $5.15 \%$ and in the E3 group it was $1.19 \%$. Comparison using the $\chi^{2}$ test revealed that the proportion of errors differed significantly between groups, $\chi^{2}(2, N=678)=17.83, p<.001, B F_{10}=65.55$. Note that $N$ stands for all trials conducted by all participants.

Surprisingly, when we looked at travel frequency, the proportion of errors in the group travelling more frequently was larger $(7.58 \%)$ than in the group travelling less frequently (3.55\%). The difference was significant, however the Bayesian analysis shows that the result is inconclusive, $\chi^{2}(1, N=678)=4.82, p=.028, B F_{10}=1.01$.

\section{Counting and montring: within-participant analyses}

Another way of looking into the finger counting and montring data was checking whether the counting-montring pattern is the same within individuals. We run this analysis for the number 1 , as this is the number, for which several differences have been reported in earlier studies (e.g. (Pika et al., 2009). Shared data allows conducting similar analyses for all other numbers. We looked whether the pattern for counting and montring is the same considering (1) hand being used; (2) finger being used; (3) exact pattern (hand and finger being used).

Hand being used. A total of 79 Tsimane' participants used the same hand for counting and montring number one, while 42 individuals used different hand. In case of British participants, a total of 44 individuals used the same hand for number one with both montring and counting, while 17 used a different hand. In case of German participants, 52 individuals used the same hand and 8 used different hand. Proportions differed significantly between groups $\chi^{2}(2, N=$ 242) $=9.16, p=.010, B F_{10}=11.39$. 
Finger being used. A total of 25 Tsimane' participants used the same finger for counting and montring number one, while 96 individuals used different finger. In case of British participants, a total of 5 individuals used the same finger for number one with both montring and counting, while 56 used a different finger. In case of German participants, 39 individuals used the same finger and 21 used different finger. Proportions differed significantly between groups $\chi^{2}(2, N$ $=242)=55.20, p<.001, B F_{10}>10^{10}$.

Same pattern being used. A total of 21 Tsimane' participants used exactly the same pattern for counting and montring number one, while 100 individuals used different pattern. In case of British participants, a total of 5 individuals used the same pattern for number one with both montring and counting, while 56 used a different pattern. In case of German participants, 35 individuals used the same finger and 25 used different pattern. Proportions differed significantly between groups $\chi^{2}(2, N=242)=48.24, p<.001, B F_{10}>10^{8}$.

\section{Discussion}

\section{Overview}

We investigated the similarities and differences in how finger counting and montring are used in Tsimane', German, and British adults. Finger counting and montring is prevalent across all three groups, as performing the tasks was easy and natural for our participants. All groups used simple one-dimensional systems (i.e., based on one-to-one correspondence) and were only using their fingers rather than onther body parts. However, groups differed in techniques (starting hand and finger, finger sequence / arrangements) used. Moreover, hand arrangements differed between finger counting and finger montring routines, with a range of configurations between groups. The thumb of the right hand was the most common starting point for counting in German and British adults. In Tsimane' no hand preference was observed and the pinkie was preferred starting finger. A range of gestures were also used in the finger montring task, where numerals 1 and 3 showed the largest variety of techniques between groups. More specifically finger counting techniques in Tsimane' were influenced by educational levels and most probably in more general, numeracy and literacy, while such a difference was not present in case of finger montring.

\section{Finger counting}

The majority of the German and British participants started counting with their righthand, which was significantly aligned with handedness. This replicates previous findings 
suggesting a preference to start counting with the dominant hand (Di Luca et al., 2006; Hohol et al., 2018; Morrissey et al., 2016; Sato, Cattaneo, Rizzolatti, \& Gallese, 2007; Sato \& Lalain, 2008), and left-handers being less likely to start with non-dominant right hand than righthanders to start with their left hand. However, still a considerable proportion of participants started with their non-dominant left hand. Importantly, there were two most prevalent finger counting patterns overall; starting from a thumb of one hand and continuing with a thumb of the other hand.

Contrary to the German and British samles, the Tsimane' sample showed no clear preference nor a link between starting hand and dominant hand. This may be due to the nature of hand dominance in indiginous groups in general. Tribal groups tend to only have strong handedness preference when using tools but not for any other activities (Marchant, McGrew, \& Eibl-Eibesfeldt, 1995). However, our handedness assessment did not show that there were more non-right-handers among Tsimane' in our sample than in German and British samples. A vast majority of Tsimane' participants started their sequence from their pinkie. Interestingly, unlike German and British samples, in Tsimane' there were four most prevalent finger counting patterns: starting from pinkie of one hand and continuing with either pinkie or thumb of the other hand. Interestingly, in case of the most prevalent pattern in Tsimane' the continuation neither followed the anatomical symmetry nor spatial continuation as number one was counted with pinkie and the sequence was continued with the thumb of the other hand. Such a pattern, to the best of our knowledge was not widely reported in other cultures / language communities (e.g. Lindemann et al., 2011). At the same time, the sequences, which were observed most commonly in Tsimane' within each hand were following the same direction (from left to right in case of participants, who started with right pinkie, or from right to left in those starting from left pinkie. This pattern deserves further investigation in future studies.

To sum up, we can conclude that Tsimane' apart from noticeable differences from both German and British samples were characterized by larger heterogeneity in finger counting routines. However, even though Tsimane' differed from German and British, the differences were confined to different order of fingers being used and different technique, but in all groups counting was similar in terms of retainig one-to-one correspondence and being limited to fingers rather than other body parts, such as other indigenous groups (Butterworth et al., 2011; Everett, 2017). These other systems have often been described as developed in response to specific environmental and cultural needs. However, the widespread character of 
the basic system can indicate it to be the most universal and efficient as adopted by multiple cultures.

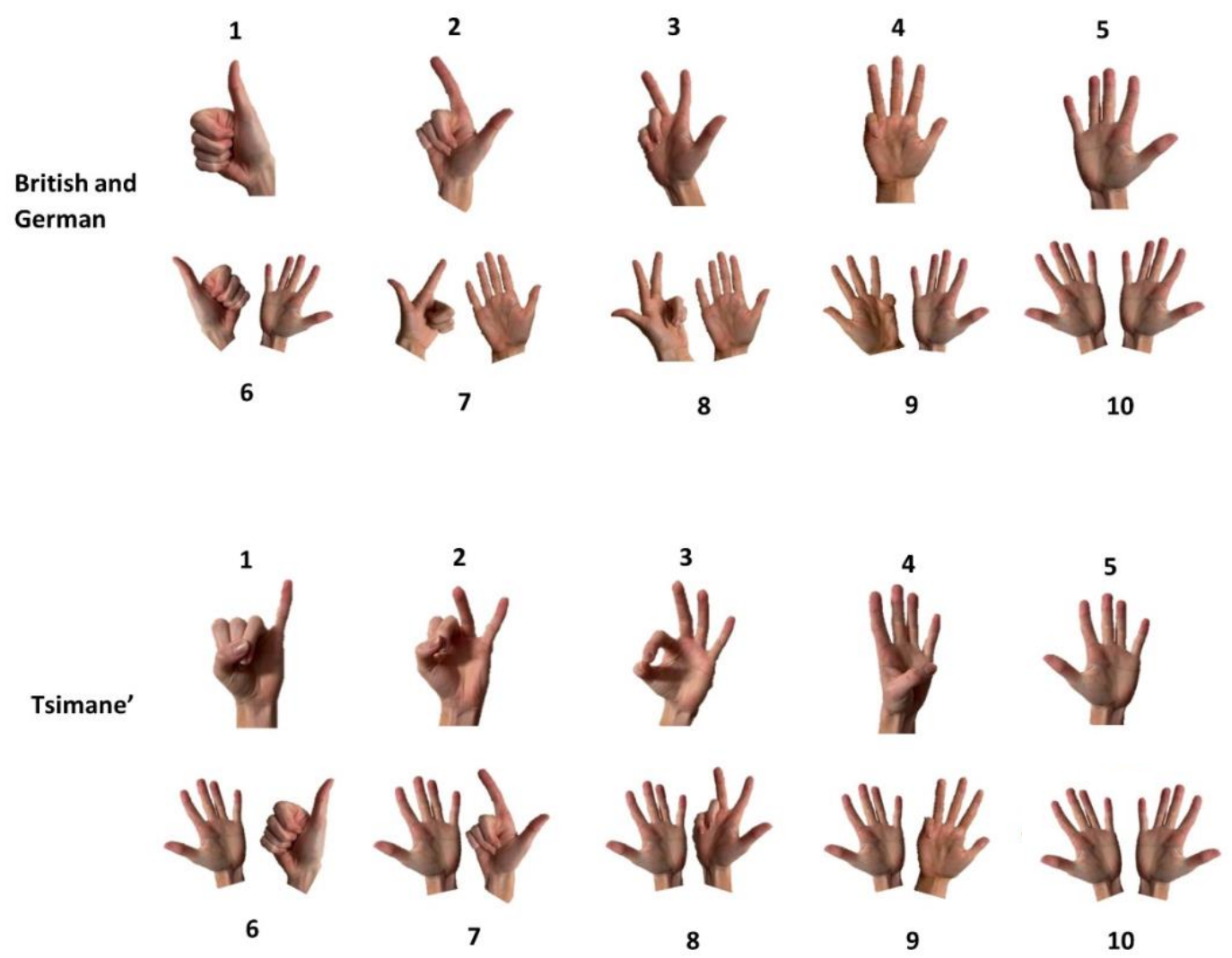

Figure 2: Most common finger counting routines used by British, German, and Tsimane'

\section{Finger Montring}

Comparing the finger counting and montring tasks, the hand arrangements, the used hand, and the used fingers often differed. Therefore, finger counting and finger montring are distinguishable in all three groups (see also Crollen, Mahe, et al., 2011) and varied between them. For number 1 the majority of British and Tsimane' participants used their right index finger - with a minority using their left index finger. This result is consistent with data on North American groups (Pika et al., 2009). Another variant was shown by some Tsimane' participants who used their left pinkie to represent 1 . The reason for this could be due to the fact that most of these participants used their pinkie to represent number 1 when they counted to 10 in the finger counting task (cardinal finger montring matched their ordinal finger counting; Wasner et al., 2015). The German participants on the other hand, mainly used their right thumb to represent this numeral (Pika et al., 2009). Additional within-subject analysis for the number one shows that only a minority of Tsimane' and British participants has used the same finger / 
hand for counting and montring number one. Such a consistency was much more prevalent across German participants. This also shows the importance for investigating both counting and montring, and their relative independence.

The montring of number 3 showed most variation between the groups (cf. Figure 3). Tsimane' started from their pinkie (similarly to their counting sequence). Germans tend to start with their thumb and at the same time, British participants used their index, middle and ring finger.

For other numbers such as 4, 6, and 9 most participants, independent of the language community, used very similar finger configuration, which most probably was the most comfortable or easiest one - due to dexterity and physical difficulties. This replicated findings of previous studies (Wasner et al., 2015). The hand-based variations of showing the numeral on either the right or left hand could be due to the handedness of the participant and their preference of starting hand.

Furthermore, for number 6, participants showed this numeral using a sub-base 5 system i.e., they presented one palm open and one finger of the other one, a common finding in German adults (Moeller et al., 2011). This system was also used for number 8 . However, for this number the variation among German participants was considerably smaller (most participans revealed one of two patterns, with pinkie and ring finger of one of the hands closed, while variation in the British and Tsimane' was larger). In the case of 9 it was necessary that one hand needs to be fully open, and either pinkie or thumb of the other hand are closed. At the same time, similar to finger counting, group differences were limited to different finger arrangements, but still the overall form (one-to-one correspondence and sticking to fingers only) were similar between groups.

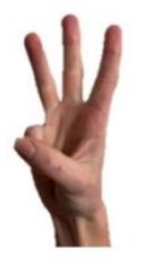

British

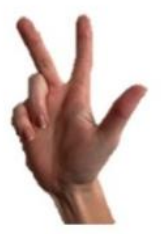

German

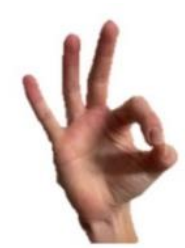

Tsimane'

Figure 3: British, German and Tsimane' finger configuration for montring number 3

Another striking observation was that there was a considerable proportion of errors in finger montring among Tsimane'. These errors might be related to their numeracy, as proportion of 
errors differed considerably depending on education level. This shows that in case of several participants, who mastered the counting sequence, the problems with cardinal numbers remained present. This seems to reflect the developmental trajectories of number concept acquisition that counting list is acquired before mastering the cardinality (Sarnecka, Goldman, \& Slusser, 2015). Other studies have shown that even though Tsimane' children follow the same developmental trajectory as children in industrialised societies, specific milestones occur at older ages (Piantadosi et al., 2014).

\section{Finger counting technique}

Group differences were observed with regards to the finger counting technique. While a vast majority of German and British participants counted by extending the fingers, half of the Tsimane' participants started with palms open, bending fingers in. Despite its scarcity in Western countries, this technique is frequent in other parts of Latin America (Closs, 1996), several parts of Africa (Zaslavsky, 1999), or Japan (Nishiyama, 2013). Nevertheless, as we only recorded the technique for a small proportion of Tsimane' participants we only mention this observation here.

\section{Outlook: Making sense out of cultural differences in finger counting}

Having demonstrated differences in finger counting and montring between Tsimane' and other groups, one may ask whether there might be some cognitive factors driving these differences as it has been suggested in previous literature (Bender \& Beller, 2011; Domahs et al., 2010). Previous studies have been able to pinpoint cognitive mechanisms underlying such differences, such as finger counting systems being used in some cultures. In case of our work, we were unable to pinpoint such mechanisms. Thety might be identified in the future studies, as the differences have now been documented. At the same time, here it might be worth asking on whether all variation in finger counting has specific cognitive underpinnings, which can be reduced to a single factor.

Functional role of the link between fingers and numbers but not specific finger-to-number link Considering these - and previously reported cultural differences, raises the question of the functional role of these differences both between groups and, more importantly, between individuals (Hohol et al., 2018). What do these differences imply, and what do they tell us about embodied numerical cognition? Reading direction-although previously suggestedcannot explain the differences, as people in predominantly left-to-right reading cultures often start counting with their right hand. In the following section we try to propose a more 
comprehensive answer by referring only to systems where the one-to-one correspondence between numbers and fingers is preserved.

What seems clear from multiple studies conducted across different cultures and countries is that fingers are used for counting (Bender \& Beller, 2011, 2012). Moreover, counting with fingers might play a functional role for numerical cognition (e.g., Andres \& Pesenti, 2015). However, given the evidence, there is no specific finger-to-number mapping that plays a specific functional role (at least in adults). However, there is some evidence that to a certain degree finger counting direction might be functionally related to Spatial-Numerical Associations (SNAs) (Pitt \& Casasanto, 2020), that is varied phenomena showing that numerical information might be associated with specific directions in space (Cipora, Schroeder, Soltanlou, \& Nuerk, 2018). We think that specific finger-to-number mappings are defined by multiple factors that may play a role for prevalence of some finger counting routines observed in different cultures, but cultural differences may not be explained by means of simple mechanisms.

\section{How can we count with our fingers?}

If we think of simple finger counting systems, in which the one-to-one correspondence between fingers and numbers is preserved and no other body parts are used in the counting sequence, the potential pool of finger counting sequences equals 10! (i.e., 3628800). If the mapping was completely random, most of us would never meet another person who uses the same sequence (and existing studies would not document two people revealing the same sequence). However, we know that this is not the case, normally we observe relatively few sequences. This is most probably determined by a few simple constraints, which seem to be followed across cultures:

(1) Starting from "outer" fingers - we do not start the sequence from a finger in the middle of the hand (i.e., middle or ring) but rather from one, which is outer: thumb, pinkie, and index (outer among the four fingers, if we treat thumb as somehow separate).

(2) Using consecutive fingers within the same hand (sometimes thumb at the end as the exception)

(3) Starting using the other hand only when we "run out" of fingers of the hand we started with. However, note that this constraint may not be the case in several African systems, which would be using both hands with symmetrical arrangements of fingers for numbers larger than 5 (e.g., number 6 would be extending three fingers of each hand, number 7, would be three and four etc. (Zaslavsky, 1999)). Nevertheless, such systems require more finger rearrangements than just adding a single finger to the current number and might be more linked to finger montring. 
These three constraints (not considering exemptions mentioned in point 3) cut down the number of possibilities - from 3628800 to 18 only: 2 (Starting hand: left vs. right) * 3 (starting finger: thumb vs. index vs. pinkie) $* 3$ (continuing finger: thumb vs. index vs. pinkie). One of these 18 patterns would be used by the majority of individuals across cultures ${ }^{4}$. We postulate several factors determining which of these sequences would be used;

Finger dexterity. Individual differences in finger dexterity, especially when it comes to the precision of operating pinkie and ring fingers, present either due to minor anatomical differences or more exercise with using specific fingers might also be noticeable at the group level (see Chui, Ng, Fong, Lin, \& Ng, 2007). Such differences might explain larger prevalence of starting with pinkie across some cultures. However, this requires further investigation.

Hand dominance. Both earlier work (Hohol et al., 2018) and our current results show that handedness affects the finger counting sequence. While among right-handers we can see a split between right- and left-starters, vast majority of left-handers are left-starters. Less established hand dominance in some cultures might lead to greater variation of the finger counting routines within these cultures (Marchant et al., 1995).

Observational learning and enculturation. Witnessing the typical finger counting sequence used within the culture can shape one's own use of fingers for counting.

Reading and writing direction. As shown in earlier studies (Cipora, Soltanlou, Reips, \& Nuerk, 2019, supplementary material; Lindemann et al., 2011), in right-to-left readers starting the finger counting with the right hand is more prevalent. Importantly, both observational learning as well as reading and writing direction could explain the increase of left-starters with increasing education in Tsimane'.

Presence of specific Spatial-Numerical Associations (SNAs) Associating numbers with specific directions in space can be liked to finger counting routine (Pitt \& Casasanto, 2020). However, the direction of the causal link between the two remains unknown. Despite individual differences in SNAs (Cipora, van Dijck, et al., 2019), the presence of the SNAs might be related not only to direction of the finger counting sequence, but even more to its stability. Hohol et al. (2018) reported that about half of the participants claim that their finger counting routine is stable. When looking at test-retest data, they observed that $75 \%$ of participants were stable in their starting hand. This corresponds well to self-reported data so that $50 \%$ are stable, and out

\footnotetext{
${ }^{4}$ In we consider patterns leading to keeping the finger pattern symmetrical we end up with 96 additional patterns: $2 *$ (starting hand) $* 3$ (starting finger for the numbers 1-5) * 16 unique patterns for counting from 6 to 10 and keeping the finger pattern symmetrical when starting with outer fingers and using consecutive fingers. Together with the 18 patterns listed in the main text, it sums up to 144, which is still dramatically fewer than number of possible permutations.
} 
of remaining $50 \%$, half of the participants happen to start with the same hand as initially, and half with the other hand. In another, yet unpublished dataset we found that out of 425 (mostly German) participants only $29 \%$ declared to always be starting their finger counting with a specific hand, and over $50 \%$ to be mostly using the same hand. At the same time, recent data (Hohol, Wołoszyn, \& Cipora, 2021) shows that there is no connection between finger counting stability and SNAs (as indexed by the SNARC effect). According to recent data of Pitt et al. (2021), Tsimane' do not show directional bias for any domain on any spatial axis, meaning that there was no left-to-right mapping of numbers. This might to some extent account for a larger variation of finger counting techniques we observed in this group. However, this requires further investigation.

Situated effects. Hohol et al. (2018) have shown that despite preferring a finger counting sequence, the flexibility to use another hand or technique in case the preferred hand is busy with holding an object or doing another task remains. This might have caused some artefacts in the existing literature (for similar arguments see Lucidi \& Thevenot, 2014; Wasner et al., 2015).

We believe that the factors listed above provide a more comprehensive account on cultural similarities and differences in finger counting routines both within and between cultures and can help guide further investigations on the topic. Importantly, not all these factors should be considered cognitive, and given variance both between and within groups, none of them seems to be highly dominant.

\section{Limitations}

Although the proportions of left-handers in our sample are representative of the general population, the absolute numbers were small and observations regarding this group should be treated with caution. However, the results were broadly consistent with those of Hohol et al. (2018). Similarly, not coding the technique in most Tsimane' prevents us from drawing conclusions. Additionally, we did not have a possibility to test non-Tsimane' Bolivians as a control group, so we cannot directly say how finger counting routines in Tsimane' match the people they encounter when visiting towns etc. This should be addressed in future studies.

Given the specificities of the tested groups, and the fact that testing Tsimane' particiants had to follow the established TSBIDSI protocol, the general circumstances of the testing were not equivalent between Tsimane' and German / British participants. At the same time, the testing protocol was to a large degree the one that was familiar to participants in each group. We acknowledge that the compensation varied between groups, however, it was in line with 
local protocols, and given that the task was neither long, tedious, nor cognitively demanding, or accuracy based, it is unlikely that this might have introduced biases in the observed results. Another potential limitation is that sample sizes and educational background differed between groups. At the same time the effects / differences between groups we observed seem to be pronounced and there seem not to be any power issue. The categorical nature of the data implies that the effects are not affected by outliers, so we do not believe that this introduces any systematic bias in the data.

When it comes to educational background, we do not aim at comparing cognitive performance between groups, but habits. At the same time, it would be good if future studies aimed at recruiting WEIRD samples with relatively low education or possibly among children who have not experience much schooling to ensure better matching.

The general cognitive skills such as working memory or IQ of the participants were not controlled for and matched between groups. As this can be considered a problem in quasiexperimental studies in general, even putting aside potential validity problems of such measures in non-WEIRD samples we think that despite being a limitation of this study, it does not undermine the conclusions. We were not judging the performance, and the task was in general probing activities the participants do in their daily lifes. Based on the general performance pattern, and high overall accuracy, the participants in all groups were familiar with finger counting and understood the instructions. There was no additional cognitive load applied either. We are also not aware of studies showing systematic differences in finger counting routines within specific cultures being dependant on demographic variables. This might well be that specific cognitive capabilities are determining the finger counting patterns, however, to the best of our knowledge such a finding was not reported in the literature, with studies showing that finger gnosia (but not specific finger counting patterns) may be linked to mathematical skills (Barrocas, Roesch, Gawrilow, \& Moeller, 2020). This is an important question on its own, and can be pursued in any culture in the future.

\section{Conclusion}

In this study we present finger counting and montring routines among nonindustrialised Tsimane' people. Even though the general picture of using fingers for counting and comminucating numbers was consistent between groups, we have also observed some differences. Apart from differences from finger counting and montring used by German and British groups we show that Tsimane' are characterized by a larger variability in their finger counting and montring. We also provide further evidence for finger counting and montring 
being distinct from each other. We outlined multiple factors such as finger dexterity, hand dominance, observational learning and encultiration, presence of SNAs, and situated influences, which might influence finger counting routines both between and within cultures. 


\section{References}

Andres, M., Olivier, E., \& Badets, A. (2008). Actions, words, and numbers: a motor contribution to semantic processing? Current directions in psychological science, 17(5), 313-317. doi:10.1111/j.1467-8721.2008.00597.x

Andres, M., \& Pesenti, M. (2015). Finger-based representation of mental arithmetic. The Oxford handbook of numerical cognition, 67-88. doi:10.1093/oxfordhb/9780199642342.013.028

Barrocas, R., Roesch, S., Gawrilow, C., \& Moeller, K. (2020). Putting a finger on numerical development-reviewing the contributions of kindergarten finger gnosis and fine motor skills to numerical abilities. Frontiers in Psychology, 11, 1012. doi:10.3389/fpsyg.2020.01012

Bender, A., \& Beller, S. (2011). Fingers as a tool for counting-naturally fixed or culturally flexible? Frontiers in Psychology, 2, 256. doi:10.3389/fpsyg.2011.00256

Bender, A., \& Beller, S. (2012). Nature and culture of finger counting: Diversity and representational effects of an embodied cognitive tool. Cognition, 124(2), 156-182. doi:10.1016/j.cognition.2012.05.005

Blackwell, A. D., Urlacher, S. S., Beheim, B., von Rueden, C., Jaeggi, A., Stieglitz, J., . . Kaplan, H. (2017). Growth references for Tsimane forager-horticulturalists of the Bolivian Amazon. American journal of physical anthropology, 162(3), 441-461. doi:10.1002/ajpa.23128

Butterworth, B. (1999). The mathematical brain: Macmillan.

Butterworth, B., Reeve, R., \& Reynolds, F. (2011). Using mental representations of space when words are unavailable: studies of enumeration and arithmetic in indigenous Australia. Journal of Cross-Cultural Psychology, 42(4), 630-638. doi:10.1177/0022022111406020

Chui, M. M., Ng, A. M., Fong, A. K., Lin, L. S., \& Ng, M. W. (2007). Differences in the fine motor performance of children in Hong Kong and the United States on the BruininksOseretsky test of motor proficiency. Hong Kong Journal of Occupational Therapy, 17(1), 1-9. doi:10.1016/S1569-1861(07)70002-5

Cipora, K., Schroeder, P. A., Soltanlou, M., \& Nuerk, H.-C. (2018). More space, better mathematics: Is space a powerful tool or a cornerstone for understanding arithmetic? In Visualizing mathematics (pp. 77-116): Springer.

Cipora, K., Soltanlou, M., Reips, U.-D., \& Nuerk, H.-C. (2019). The SNARC and MARC effects measured online: Large-scale assessment methods in flexible cognitive effects. Behavior research methods, 51(4), 1676-1692. doi:10.3758/s13428-019-01213-5

Cipora, K., van Dijck, J.-P., Georges, C., Masson, N., Goebel, S., Willmes, K., . . Nuerk, H.C. (2019). A Minority pulls the sample mean: on the individual prevalence of robust group-level cognitive phenomena-the instance of the SNARC effect. Psyarxiv. doi:10.31234/osf.io/bwyr3

Closs, M. P. (1996). Native american mathematics: University of Texas Press.

Crollen, V., Mahe, R., Collignon, O., \& Seron, X. (2011). The role of vision in the development of finger-number interactions: Finger-counting and finger-montring in blind children. Journal of experimental child psychology, 109(4), 525-539. doi:10.1016/j.jecp.2011.03.011

Crollen, V., Seron, X., \& Noël, M.-P. (2011). Is finger-counting necessary for the development of arithmetic abilities? Frontiers in Psychology, 2, 242. doi:10.3389/fpsyg.2011.00242 
Di Luca, S., Granà, A., Semenza, C., Seron, X., \& Pesenti, M. (2006). Finger-digit compatibility in Arabic numeral processing. Quarterly Journal of Experimental Psychology, 59(9), 1648-1663. doi:10.1080/17470210500256839

Di Luca, S., \& Pesenti, M. (2008). Masked priming effect with canonical finger numeral configurations. Experimental Brain Research, 185(1), 27-39. doi:10.1007/s00221007-1132-8

Di Luca, S., \& Pesenti, M. (2011). Finger numeral representations: more than just another symbolic code. Frontiers in Psychology, 2, 272. doi:10.3389/fpsyg.2011.00272

Domahs, F., Moeller, K., Huber, S., Willmes, K., \& Nuerk, H.-C. (2010). Embodied numerosity: implicit hand-based representations influence symbolic number processing across cultures. Cognition, 116(2), 251-266. doi:10.1016/j.cognition.2010.05.007

Everett, C. (2017). Numbers and the Making of Us: Harvard University Press.

Ferrigno, S., Jara-Ettinger, J., Piantadosi, S. T., \& Cantlon, J. F. (2017). Universal and uniquely human factors in spontaneous number perception. Nature communications, 8(1), 1-10. doi:10.1038/ncomms 13968

Fischer, M. H. (2008). Finger counting habits modulate spatial-numerical associations. cortex, 44(4), 386-392. doi:10.1016/j.cortex.2007.08.004

Fischer, M. H., Kaufmann, L., \& Domahs, F. (2012). Finger counting and numerical cognition. Frontiers in Psychology, 3, 108. doi:10.3389/fpsyg.2012.00108

Gashaj, V., Oberer, N., Mast, F. W., \& Roebers, C. M. (2019). Individual differences in basic numerical skills: The role of executive functions and motor skills. Journal of experimental child psychology, 182, 187-195. doi:10.1016/j.jecp.2019.01.021

Gibson, E., Jara-Ettinger, J., Levy, R., \& Piantadosi, S. (2017). The use of a computer display exaggerates the connection between education and approximate number ability in remote populations. Open Mind, 2(1), 37-46. doi:10.1162/opmi_a_00016

Göbel, S. M., Shaki, S., \& Fischer, M. H. (2011). The cultural number line: a review of cultural and linguistic influences on the development of number processing. Journal of Cross-Cultural Psychology, 42(4), 543-565. doi:10.1177/0022022111406251

Godoy, R., \& Jacobson, M. (1999). Covariates of private time preference: A pilot study among the Tsimane'Indians of the Bolivian rain forest. Evolution and human behavior, 20(4), 249-256. doi:10.1016/S1090-5138(99)00009-4

Godoy, R., Jacobson, M., \& Wilkie, D. (1998). Strategies of rain-forest dwellers against misfortunes: the Tsimane'Indians of Bolivia. Ethnology, 55-69. doi:10.2307/3773848

Henrich, J., Heine, S. J., \& Norenzayan, A. (2010). Most people are not WEIRD. Nature, 466(7302), 29-29. doi:10.1038/466029a

Hohol, M., Wołoszyn, K., \& Cipora, K. (2021). No fingers, no SNARC? Spatial-Numerical Associations and temporal stability of finger counting. Psyarxiv. doi:10.31234/osf.io/23mya

Hohol, M., Wołoszyn, K., Nuerk, H.-C., \& Cipora, K. (2018). A large-scale survey on finger counting routines, their temporal stability and flexibility in educated adults. PeerJ, 6 , e5878. doi: $10.7717 /$ peerj. 5878

Huanca, T. (2008). Tsimane'Oral Tradition, Landscape, and Identity in the Tropical Forest TAPS: Research: Publications: Books: Tsimane'Oral Tradition. In.

Ifrah, G. (1985). From one to zero: A universal history of numbers: Viking Adult.

Jara-Ettinger, J., Gibson, E., Kidd, C., \& Piantadosi, S. (2016). Native Amazonian children forego egalitarianism in merit-based tasks when they learn to count. Developmental science, 19(6), 1104-1110. doi:10.1111/desc.12351 
Lindemann, O., Alipour, A., \& Fischer, M. H. (2011). Finger counting habits in middle eastern and western individuals: an online survey. Journal of Cross-Cultural Psychology, 42(4), 566-578. doi:10.1177/0022022111406254

Love, J., Selker, J., \& Dropmann, D. (2021). jsq: Bayesian Methods. R package version 0.9.0.

Lucidi, A., \& Thevenot, C. (2014). Do not count on me to imagine how I act: behavior contradicts questionnaire responses in the assessment of finger counting habits. Behavior research methods, 46(4), 1079-1087. doi:10.3758/s13428-014-0447-1

Marchant, L. F., McGrew, W. C., \& Eibl-Eibesfeldt, I. (1995). Is human handedness universal? Ethological analyses from three traditional cultures. Ethology, 101(3), 239258. doi:10.1111/j.1439-0310.1995.tb00362.x

Moeller, K., Martignon, L., Wessolowski, S., Engel, J., \& Nuerk, H.-C. (2011). Effects of finger counting on numerical development-the opposing views of neurocognition and mathematics education. Frontiers in Psychology, 2, 328. doi:10.3389/fpsyg.2011.00328

Morrissey, K. R., Liu, M., Kang, J., Hallett, D., \& Wang, Q. (2016). Cross-cultural and intracultural differences in finger-counting habits and number magnitude processing: embodied numerosity in Canadian and Chinese university students. Journal of Numerical Cognition, 2(1), 1-19. doi:10.5964/jnc.v2i1.14

Nishiyama, Y. (2013). Counting with the fingers. International Journal of Pure and Applied Mathematics, 85(5), 859-868. doi:10.12732/ijpam.v85i5.4

Overmann, K. A. (2021). Finger-counting in the Upper Palaeolithic. Rock Art Research, 31(1), 63-80. doi:10.31235/osf.io/wgbe5

Piantadosi, S. T., Jara-Ettinger, J., \& Gibson, E. (2014). Children's learning of number words in an indigenous farming-foraging group. Developmental science, 17(4), 553-563. doi:10.1111/desc. 12078

Pika, S., Nicoladis, E., \& Marentette, P. (2009). How to order a beer: cultural differences in the use of conventional gestures for numbers. Journal of Cross-Cultural Psychology, 40(1), 70-80. doi:10.1177/0022022108326197

Pitt, B., \& Casasanto, D. (2020). The correlations in experience principle: How culture shapes concepts of time and number. Journal of Experimental Psychology: General, 149(6), 1048. doi:10.1037/xge0000696

Pitt, B., Ferrigno, S., Cantlon, J., Casasanto, D., Gibson, E., \& Piantadosi, S. T. (2021). Spatial concepts of number, size, and time in an indigenous culture. Science Advances, 7(33). doi:10.1126/sciadv.abg4141

Roesch, S., \& Moeller, K. (2015). Considering digits in a current model of numerical development. Frontiers in human neuroscience, 8, 1062. doi:10.3389/fnhum.2014.01062

Roullion, A. (2006). Au Gravettien, dans la grotte Cosquer (Marseille, Bouches-du-Rhône), l'Homme at-il compté sur ses doigts? During the Gravettian, in the Cosquer cave (Marseille, Bouches-du-Rhône), has the Man counted on his fingers? L'Anthropologie, 110(4), 500-509. doi:10.1016/j.anthro.2006.07.003

Sarnecka, B. W., Goldman, M. C., \& Slusser, E. B. (2015). How counting leads to children's first representations of exact, large numbers. Oxford, UK: Oxford University Press.

Sato, M., Cattaneo, L., Rizzolatti, G., \& Gallese, V. (2007). Numbers within our hands: modulation of corticospinal excitability of hand muscles during numerical judgment. Journal of cognitive neuroscience, 19(4), 684-693. doi:10.1016/j.cortex.2007.08.005

Sato, M., \& Lalain, M. (2008). On the relationship between handedness and hand-digit mapping in finger counting. cortex, 44(4), 393-399. doi:10.1016/j.cortex.2007.08.005

Saxe, G. B., \& Esmonde, I. (2012). Cultural development of mathematical ideas: Papua New Guinea studies: Cambridge University Press. 
Selker, J., Love, J., \& Dropmann, D. (2020). jmv: The'jamovi'Analyses. R package version 1.2. 23 .

Sihler, A. L. (2008). New comparative grammar of Greek and Latin: Oxford University Press.

van der Linden, D., Dunkel, C. S., Figueredo, A. J., Gurven, M., von Rueden, C., \& Woodley of Menie, M. A. (2018). How universal is the general factor of personality? An analysis of the Big Five in forager farmers of the Bolivian Amazon. Journal of CrossCultural Psychology, 49(7), 1081-1097. doi:10.1177/0022022118774925

Wasner, M., Moeller, K., Fischer, M. H., \& Nuerk, H.-C. (2015). Related but not the same: Ordinality, cardinality and 1-to-1 correspondence in finger-based numerical representations. Journal of Cognitive Psychology, 27(4), 426-441. doi:10.1080/20445911.2014.964719

Wickham, H., François, R., Henry, L., \& Müller, K. (2020). RStudio, dplyr: A Grammar of Data Manipulation.

Wiese, H. (2003). Numbers, language, and the human mind: Cambridge University Press.

Zago, L., \& Badets, A. (2016). What is the role of manual preference in hand-digit mapping during finger counting? A study in a large sample of right-and left-handers. Perception, 45(1-2), 125-135. doi:10.1177/0301006615602628

Zaslavsky, C. (1999). Africa counts: Number and pattern in African cultures: Chicago Review Press. 


\section{Supplementary Material}

Table S1. Montring, Education, and Travel Frequency

\begin{tabular}{lllllccc} 
& \multicolumn{3}{c}{ Education } & \multicolumn{3}{c}{ Travel Frequency } \\
\hline \multirow{2}{*}{ Number } & \multicolumn{1}{c}{$\chi^{2}$ Test* } & Montring Errors & $\chi^{2}$ Test & Montring Errors \\
\hline & & E1 & E2 & E3 & & T1 & T2 \\
3 & $\chi^{2}(12, N=112)=8.53$ & 1 & 0 & 0 & $\chi^{2}(6, N=112)=9.73$ & 0 & 1 \\
4 & $\chi^{2}(10, N=113)=13.70$ & 0 & 0 & 0 & $\chi^{2}(5, N=113)=3.75$ & 0 & 0 \\
6 & $\chi^{2}(6, N=112)=8.54$ & 0 & 1 & 0 & $\chi^{2}(3, N=112)=4.13$ & 0 & 1 \\
8 & $\chi^{2}(12, N=104)=10.66$ & 5 & 4 & 0 & $\chi^{2}(6, N=104)=6.10$ & 2 & 7 \\
9 & $\chi^{2}(12, N=101)=9.59$ & 8 & 4 & 0 & $\chi^{2}(6, N=101)=3.03$ & 3 & 9 \\
\hline
\end{tabular}

Note. E1 $=$ no schooling; E2 $=1-5$ years of education; E3 $=$ more than 5 years of education. T1 $=$ travel less than twice a month; $\mathrm{T} 2=$ travel twice a month and more. *all reported $\chi 2$ tests were non-significant. Except the comparison of education in case of the number 9 (see main text), the Bayesian analysis supported the null hypothesis model or remained inconclusive. 